\title{
Diagnosis and Management of IUGR in Pregnancy Complicated by Type 1 Diabetes Mellitus
}

\author{
Pawel Gutaj $^{1}$ • Ewa Wender-Ozegowska ${ }^{1}$
}

Published online: 16 March 2016

(C) The Author(s) 2016. This article is published with open access at Springerlink.com

\begin{abstract}
This review discusses available literature on the diagnosis and management of intrauterine growth restriction (IUGR) in women with type 1 diabetes. IUGR is diagnosed when ultrasound-estimated fetal weight is below the 10th percentile for gestational age. IUGR diagnosis implies a pathologic process behind low fetal weight. IUGR in pregnancy complicated by type 1 diabetes is usually caused by placental dysfunction related to maternal vasculopathy. Prevention of IUGR should ideally start before pregnancy. Strict glycemic control and intensive treatment of nephropathy and hypertension are essential. Low-dose aspirin initiated before 16 gestational weeks can also reduce IUGR risk in women with vasculopathy. Umbilical and uterine artery Doppler studies can guide diagnosis and surveillance of fetuses with IUGR. Decisions regarding the timing of delivery should be based on assessment of umbilical artery Doppler. The risk of prematurity and impaired fetal lung maturation should always be considered, especially in fetuses younger than 32 weeks.
\end{abstract}

Keywords Intrauterine growth restriction · IUGR - Small for gestational age $\cdot$ SGA $\cdot$ Type 1 diabetes · Vasculopathy

This article is part of the Topical Collection on Diabetes and Pregnancy

Paweł Gutaj

pgutaj@o2.pl

Ewa Wender-Ozegowska

ewaoz@post.pl

1 Department of Obstetrics and Women's Diseases, Poznan University of Medical Sciences, Polna 33, 60-535 Poznan, Poland

\section{Introduction}

Fetal growth is a result of complex interactions between the genetic growth potential of the fetus and the impact of the maternal intrauterine environment. Causes of intrauterine growth restriction (IUGR) are generally classified as maternal, fetal, or placental. However, such distinction is rather theoretical, as these factors often overlap each other.

Impaired fetal growth not only results in elevated risk for short-term complications, with intrauterine fetal death being the most severe, but low birth weight also predisposes the child to cardiovascular disease and type 2 diabetes mellitus in the future $[1,2]$.

IUGR is a common cause of iatrogenic prematurity, as a manifestation of ischemic placental disease - a syndrome of placental insufficiency found in several pregnancies terminated prematurely due to fetal conditions.

IUGR is diagnosed when ultrasound-estimated fetal weight is below the 10th percentile for gestational age. A diagnosis of IUGR implies a pathologic growth restriction responsible for low fetal weight [3-5]. Small for gestational age (SGA) is another term that defines a fetus with an estimated weight below the 10th percentile; however, the term SGA does not imply a pathological condition causing low fetal weight. Approximately $70 \%$ of SGA fetuses are constitutionally small, meaning they are small but healthy. The remaining are fetuses with IUGR, which are at high risk for perinatal complications and therefore require more intensive surveillance $\left[6^{\bullet}\right]$.

Fetal growth abnormalities are frequently seen in diabetic pregnancy, with large for gestational age (LGA) being the most typical one. LGA has been widely studied and seems to be associated most frequently with maternal hyperglycemia, although other factors such as gestational weight gain and maternal lipids may also play a role [7-12]. However, 
diabetic pregnancy can also be associated with restricted fetal growth. Long-standing and poorly controlled type 1 diabetes represents a wide spectrum of clinical conditions, mostly related to diabetic vasculopathy. Since the placenta is an organ composed almost entirely of vessels, maternal vasculopathy can be linked to placental dysfunction and subsequent fetal growth restriction [13-15].

The incidence of type 1 diabetes is rising at an alarming rate, particularly in younger age groups. Although disease outcomes have been improving over the past decades, resulting in a reduction in total mortality, these beneficial trends start to disappear for the incidence of nephropathy and proliferative retinopathy at 20 years of diabetes duration [16]. Thus, it may be expected that an increasing number of women with diabetic vascular disease are now, or soon will be, entering childbearing age. IUGR could therefore become one of the major pregnancy complications exacerbated by type 1 diabetes.

This review discusses available literature on the diagnosis and management of IUGR in women with type 1 diabetes. However, data on IUGR in type 1 diabetic pregnancy is limited and there are no randomized controlled trials on the management of IUGR in this group of patients. This significantly limits the strength of the evidence. Therefore, we discuss existing guidelines for a normal population in the context of diabetic pregnancy and focus on expert opinion as well as our personal experience regarding the management of diabetic pregnancy.

\section{Pathogenesis of IUGR in Pregnancy Complicated by Type 1 Diabetes - the Key Role of Diabetic Vasculopathy}

Many factors can potentially interfere with genetically predetermined fetal growth causing its restriction. In this section, we provide an overview of maternal and placental factors related to diabetes that cause IUGR.

The incidence of type 1 diabetes peaks in 10-14 year olds and is rising at an alarming rate worldwide, particularly in younger age groups (0-4 years) [17]. Many of these girls are reaching childbearing age as women with long-standing diabetes, frequently complicated by vasculopathy.

Metabolic alterations associated with diabetes impact the development and function of utero-placental and fetoplacental units from the beginning of pregnancy. Fetal growth in type 1 diabetic pregnancy might be altered in two opposing ways. Maternal hyperglycemia stimulates fetal overgrowth, while maternal vasculopathy can be associated with placental insufficiency leading to altered nutrient transport and subsequent IUGR.

\section{Histopathological Studies}

Several histopathological changes can occur in the placenta in response to a diabetic environment [18]. Although some of them are not specific for the diabetic placenta, maternal arterial malperfusion, distal villus maldevelopment, and fetal vasculitis might be considered characteristics for diabetic placentas. Maternal arterial malperfusion occurs as a consequence of inadequate and incomplete invasion of the trophoblast and deficient spiral artery transformation [19]. Moreover, unremodeled uterine arteries can additionally present features of decidual vasculopathy. It has been previously shown that almost a quarter of type 1 diabetic placentas harbored decidual vasculopathy [20]. These pathological changes are associated with IUGR, particularly in preeclamptic women [19, 21]. Women with type 1 diabetes are at substantially higher risk of preeclampsia. Furthermore, the risk increases with longer diabetes duration, with the highest rates reported in women with diabetic nephropathy, placing them at the highest risk of developing IUGR [22]. Distal villus maldevelopment is a term representing a group of histopathological findings including distal villous hypoplasia, distal villous immaturity, chorangiosis, and dysmorphic villi. These changes are associated with the reduction of functional villus surface area and can be observed in a significant proportion of IUGR placentas $[23,24]$. Fetal vasculitis is a common histopathological finding in diabetic placentas and might be a marker of fetal inflammatory response $[20,25]$. The role of fetal vasculitis in IUGR remains unknown; however, some authors reported an association between different markers of inflammatory state and IUGR [26, 27].

\section{Biochemical Studies}

Abnormalities in placental angiogenesis due to maternal microangiopathy have their roots early in pregnancy. It has been demonstrated that placental growth factor (PlGF) concentration dynamics is impaired in women with type 1 diabetes whose fetuses later develop SGA. While PlGF serum concentration doubled between the first and second trimesters in women with type 1 diabetes who delivered newborns with normal growth, there was no physiological increase in PlGF in the SGA group. Importantly, the majority of women from the SGA group had some form of diabetic vasculopathy [13]. Other interesting findings come from a study on markers of endothelial dysfunction. Zawiejska et al. observed elevated maternal serum concentration of vascular cell adhesion molecule-1 (VCAM-1) in early gestation and low levels in late gestation in women with type 1 diabetes who gave birth to SGA newborns. The authors hypothesized that observed changes may be a manifestation of endothelial injury in early gestation followed by placental dysfunction in late gestation. Early vascular dysfunction was also proposed as a major factor involved 
in preeclampsia in women with type 1 diabetes. The authors of this study demonstrated significantly increased first trimester serum levels of VCAM-1 and intercellular adhesion molecule-1 (ICAM-1) in the preeclamptic group [28].

\section{Doppler Ultrasound Findings}

Although placental histopathological findings provide substantial and clinically relevant information, these data are always retrospective and represent pathological processes and adaptations that have been taking place sometimes many weeks before delivery. Doppler ultrasound enables the realtime assessment of placental and fetal circulation and has been widely used in the monitoring of high-risk pregnancies. Moreover, abnormal umbilical and uterine Doppler velocity waveforms in IUGR have been correlated with abnormal placental pathology [29-31]. Efficient utero-placental and fetoplacental circulation is crucial for the maintenance of physiological fetal growth. Pietryga et al. demonstrated significantly increased uterine artery vascular impedance in pregnant women with type 1 diabetes with vasculopathy [32]. Furthermore, increasing uterine artery scores were associated with an increasing proportion of SGA newborns. In this study, abnormal uterine Doppler was almost exclusively seen in women with classes R/F retinopathy and nephropathy, according to White [33]. Women with uncomplicated diabetes or isolated retinopathy had relatively normal Doppler results. The proportion of abnormal flow in umbilical arteries was significantly lower and unrelated to vasculopathy. This suggests that impairment of utero-placental circulation is a primary pathology in the placentas of women with severe vasculopathy and changes in the umbilical artery may occur later as an adaptation to reduced utero-placental perfusion. Impairment of placental function in diabetic vasculopathy has been directly confirmed in a study by Salvesen et al. It was conducted in a cohort of 41 women with type 1 diabetes undergoing cordocentesis up to $24 \mathrm{~h}$ before delivery. The authors found that all fetuses of women with nephropathy and hypertension had significantly lower umbilical vein $\mathrm{pH}$ than the normal mean for gestation $(<5$ th percentile) [34].

\section{Diagnosis of IUGR}

\section{Diagnostic Approach}

Because IUGR is associated with unfavorable perinatal outcomes and therefore has a strong impact on the management of pregnancy, it should be clearly distinguished from constitutional small for gestational age (cSGA). The most widely used definition of IUGR is based on the estimated fetal weight (EFW) below the 10th percentile [3-5]. However, according to the Royal College of Obstetricians and Gynecologists
(RCOG), the diagnosis of IUGR can also be made on the basis of fetal abdominal circumference (AC) below the 10th percentile [4]. The brief summary of diagnostic criteria as well as important aspects on the management of IUGR is summarized in Table 1. It should be mentioned that percentile charts used for these determinations were developed based on the healthy population. Persson et al., in their large population-based cohort study, showed that distribution of birth weight (BW) of offspring born to the mothers with type 1 diabetes was significantly wider and shifted to the right of the normal reference. Moreover, newborns of the mothers with type 1 diabetes had higher mean BW despite significantly lower mean gestational age (GA) at birth [35]. Similar findings have been demonstrated by others in smaller cohorts [36, 37]. However, Howart et al. showed that such distribution is rather a characteristic for uncomplicated type 1 diabetic pregnancy. These authors demonstrated that customized BW percentiles of newborns of women with vascular disease (defined as the presence of at least one of the following: diabetic retinopathy, diabetic nephropathy, or preexisting hypertension) were bimodally distributed [38]. The authors concluded that coexistence of diabetes and vascular disease produces an even more unfavorable intrauterine environment, which may falsely "normalize" the growth of fetuses destined to develop macrosomia. One may suggest that among these fetuses, some could be growth restricted due to impaired placental function and "normalized" by maternal hyperglycemia. These facts raise the question whether the diagnosis of IUGR in women with type 1 diabetes is appropriate using charts based on a healthy population. This question still remains open, as there is no evidence in the literature to support different diagnostic approaches. Therefore, the diagnosis of IUGR in women with type 1 diabetes should be based on the same criteria as for the reference population. Nonetheless, special attention should be paid to fetuses with borderline estimated fetal weight, as the risk for false negative diagnosis may be increased due to changes in their weight distribution. In suspicious or questionable cases, uterine and umbilical artery Doppler ultrasound may provide useful diagnostic information, especially when IUGR is of placental origin in women with diabetic vasculopathy [32, 39].

\section{Importance of Pregnancy Dating}

Accurate pregnancy dating is essential for the diagnosis of IUGR. However, estimation of GA based on the last menstrual period (LMP) might be inadequate in a significant proportion of women with type 1 diabetes due to frequent menstrual irregularities [40, 41].

Measurement of crown-rump length (CRL), optimally between weeks 9 and 13 of gestation, provides the most reliable basis for further evaluation of fetal growth and should be precisely documented in women with type 1 diabetes $[42,43]$. 


\section{Early Detection of Women at Risk}

Detection of patients at risk of IUGR should ideally start early in the pregnancy. In addition to traditional medical and obstetric risk factors for IUGR, women with type 1 diabetes should be screened for vascular disease, primarily nephropathy $[14$, 38]. Notably, not only overt nephropathy but also microalbuminuria has been associated with IUGR [15]. Despite the known association between diabetic retinopathy and adverse pregnancy outcomes, there are no studies directly linking diabetic retinopathy to IUGR $[44,45]$. One study reported no difference in the incidence of fetal growth restriction between women with microvascular complications (retinopathy and nephropathy, analyzed together) and those without them [46]. This might be attributed to the significant improvements in the quality of care in pregnant women with type 1 diabetes, particularly intensification of antihypertensive treatment [47•].

Diabetes, especially when uncontrolled early in pregnancy, increases the risk of fetal structural anomalies which may also manifest as impaired fetal growth [48, 49]. IUGR fetuses should therefore undergo detailed anatomy scans involving echocardiography.

\section{Management of IUGR}

IUGR increases the risk of adverse pregnancy outcomes including preterm delivery, intrauterine fetal death (IUFD), neonatal death, low APGAR scores, and abnormal neurodevelopment of the child [50,51]. Thorough prenatal surveillance and appropriate timing of delivery can reduce the risk of perinatal mortality and morbidity in women with type 1 diabetes. Women with pregnancies complicated by type 1 diabetes and IUGR should be managed by maternal-fetal medicine specialists experienced in the field of diabetic pregnancy at a perinatal center with access to a neonatal intensive care unit.

\section{Ultrasound Surveillance}

Ultrasound biometry is essential for the diagnosis of IUGR due to its superiority in clinical weight estimation using fundal height measurements. Therefore, it seems reasonable to perform ultrasound biometry in all women with risk factors for fetal growth abnormalities, including those with diabetes. IUGR associated with diabetic vasculopathy and resultant placental insufficiency is typically asymmetric and does not manifest before the third trimester [52]. Symmetric growth restriction diagnosed earlier in the second trimester raises the suspicion of genetic disorders or congenital malformations and standard management should be offered to these women. 
Subsequent serial ultrasound measurements provide further information on fetal growth pattern, which might be altered in women with type 1 diabetes [52]. The optimal intervals between scans have not been determined and should be based on individual clinical judgment. However, it is suggested that repeated measurements should be performed at least 3 weeks apart to reduce the possibility of a false positive diagnosis [3, 4]. There is no evidence to support more frequent fetal biometry in women with type 1 diabetes and IUGR.

Amniotic fluid volume (AFV) assessment is a routine part of ultrasound scanning during pregnancy. Although, oligohydramnios is associated with SGA and perinatal mortality, its predictive value for individual risk for the outcome is poor [53]. Thus, AFV measurement should not be used as the only method of monitoring IUGR fetuses. However, in conjunction with Doppler studies or as a part of biophysical profiles (BPP), it can provide more clinically relevant information on fetal well-being. AFV can be assessed by subjective (based on the examiner's visual assessment) and objective (amniotic fluid index [AFI] and single deepest pocket [SDP]) methods. It is recommended to measure AFV objectively when either subjective measurements suggest decreased AFV or in patients with higher risk of IUGR. Comparison of AFI and SDP revealed that AFI was associated with higher rates of false positive diagnosis of oligohydramnios which may lead to unnecessary labor induction [54]. Thus, SDP measurement may be preferred over AFI, especially in preterm pregnancies.

Although there are no randomized trials examining the utility of ultrasound Doppler surveillance in pregnancy complicated by type 1 diabetes, existing evidence from observational studies support this method as the best for detection of fetal distress in high-risk pregnancies, including IUGR.

Doppler ultrasound directly reflects impairment of uteroplacental and feto-placental circulation which is, in the case of IUGR, related to maternal vasculopathy $[32,39]$. In addition to its essential role in determining the cause of fetal growth restriction (placental insufficiency vs. constitutional SGA/ other causes), umbilical Doppler velocimetry has been widely recommended as a main method for the follow-up of IUGR fetuses [3-5]. In pregnancy complicated by type 1 diabetes, umbilical artery Doppler velocimetry was found to be superior to non-stress test and BPP in identifying fetuses at risk of adverse outcome [55]. In normal circumstances, in the second part of pregnancy, umbilical and uterine arteries are lowresistance vessels. In cases of placental dysfunction, both vessels can present features of increasing flow resistance. These alterations are manifested in Doppler as increasing pulsation index (PI), the presence of end-diastolic notches in uterine arteries, and increasing PI in umbilical arteries. Increasing resistance in umbilical arteries can cause severe hemodynamic changes which can be further manifested as absent enddiastolic velocity (AEDV) and reversed end-diastolic velocity (REDV).

\section{Biophysical Profile (BPP) and Fetal Heart Rate Monitoring}

The number of studies on the utility of BPP and fetal heart rate monitoring (FHRM) in pregnancy complicated by type 1 diabetes is limited $[34,55,56]$. It was shown that both BPP and FHRM had lower predictive value of adverse pregnancy outcomes including fetal growth restriction than umbilical artery Doppler [55] and their results were not predictive for fetal acidemia [34]. The low predictive value of abnormal BPP has been confirmed in a prospective study using multiple BPP from 28 weeks until delivery [56]. Nonetheless, the high predictive value of normal BPP makes it a more valuable tool for surveillance of fetuses at high risk of IUFD, including these with IUGR. However, it should be remembered that diabetes may have an impact on the results of BPP as maternal hyperglycemia was found to increase both fetal respiratory movements and AFV [57, 58]. Furthermore, sustained hyperglycemia $(>120 \mathrm{mg} / \mathrm{dL})$ was associated with a decrease in fetal movements in healthy women [59]. No significant changes in fetal body and respiratory movements were observed during hypoglycemia [60].

\section{Maternal Perception of Fetal Movements}

Decreased fetal movements (DFM) perception in the third trimester has been associated with increased rates of IUGR, emergency cesarean section (CS), and IUFD [61]. There are no separate guidelines on the management of DFM in women with type 1 diabetes. Nonetheless, reports of DFM should always prompt further investigation, including confirmation of fetal heart rate and weight estimation by ultrasound. If the fetus is found to be IUGR, umbilical and uterine Doppler should be considered due to the high risk of placental dysfunction, primarily in women with vasculopathy [32].

\section{Diet and Physical Activity}

There is no evidence that changes in diet or supplement use can prevent or treat IUGR. Thus, individualized medical nutrition therapy (IMT) for pregnant women with type 1 diabetes is indicated. IMT is aimed at achieving glycemic targets and maintaining appropriate gestational weight gain [62].

There is no evidence to recommend bed rest as a method of either prevention or treatment of IUGR [63]. However, strenuous physical activity should be avoided due to risk of hypoglycemia.

\section{Glycemic Targets and IUGR}

There is no evidence to support the use of individualized glycemic targets in pregnant women with type 1 diabetes and IUGR. Nonetheless, Langer et al. showed in women with 
gestational diabetes mellitus (GDM) that had low levels of mean glycemia throughout pregnancy $(<86 \mathrm{mg} / \mathrm{dL})$ were associated with a significant increase in SGA in comparison to healthy controls (20 vs. $11 \%$ ) [64]. To the authors' knowledge, there are no similar studies in women with type 1 diabetes and IUGR. However, it could be hypothesized that too strict glycemic control in women with already impaired placental function and diminished nutrient transport to the fetus could cause further restriction of fetal growth with all associated complications [65].

\section{Antenatal Corticosteroids}

IUGR is associated with significantly increased rates of preterm deliveries, primarily iatrogenic. Diabetes is not considered a contraindication for the administration of corticosteroids for fetal lung maturation, but this procedure should be precisely planned with increased dosages of insulin [66]. There is no full consensus on the time window when corticosteroids should be given in pregnancies complicated by IUGR. The American College of Obstetricians and Gynecologists (ACOG) and the Society of Obstetricians and Gynaecologists (SOGC) recommend a single course of corticosteroids when there is a significant risk of delivery $<34$ weeks of gestation [3,5]. The RCOG recommends that in pregnancy with fetal growth restriction and anticipated preterm delivery, a single course of corticosteroids should be given until $35+6$ weeks of gestation [4].

However, the strongest evidence exists for supporting the use of corticosteroids until $34+6$ weeks [67]. Furthermore, bearing in mind that high doses of potent corticosteroids (betamethasone or dexamethasone) can significantly deteriorate maternal glycemic control, it seems reasonable to recommend stimulation for fetal lung maturation until $34+6$ weeks in women with type 1 diabetes. Treatment with corticosteroids should be followed by appropriate insulin dose adjustment and frequent glycemic monitoring [68, 69].

\section{Timing and Mode of Delivery}

The aim of intensive surveillance of the fetus with IUGR is to detect the safest time for delivery. This is often difficult for even the most experienced obstetrician, especially in cases of fetal prematurity. Thus, several factors should be taken into careful consideration while making a decision on timing and mode of delivery for IUGR fetus. Management always depends on the etiology of IUGR. In women with type 1 diabetes, IUGR is typically of placental origin. Undoubtedly, this is the most difficult to manage and most unpredictable type of IUGR, especially in women with nephropathy and preeclampsia. In such cases, the decision on timing of delivery is often based on either signs of acute fetal distress, deterioration of maternal state, or both. There are several tools for fetal surveillance; however, only some have been proven to predict neonatal outcomes. None of them have been studied exclusively in women with type 1 diabetes and IUGR in the context of timing of delivery. Doppler velocimetry is considered the most sensitive method to detect risk of fetal death. Although several vessels can be examined during this procedure, only umbilical artery velocimetry has been found to decrease the likelihood of perinatal deaths in IUGR [70]. Therefore, it is recommended to base the decision regarding timing of delivery on the results of umbilical artery Doppler studies (absent or reversed end-diastolic velocity [AREDV]) $[3,5,70]$. Nonetheless, GA is the major determinant of fetal survival in IUGR diagnosed before 33 weeks [71]. RCOG recommends that in fetuses with AREDV detected prior to 32 weeks, delivery should be considered when either ductus venosus (DV) abnormalities or umbilical vein (UV) pulsation is found [4].

The IUGR itself is not an indication for CS. However, a significant number of IUGR fetuses is delivered via urgent CS due to acute signs of fetal distress demonstrated either before the onset of spontaneous contractions (umbilical artery AREDV, UV pulsation, DV abnormalities) or intrapartum (late or variable decelerations, loss of fetal heart rate variability). IUGR of placental origin is a manifestation of chronic fetal distress, and the fetus may already be acidotic. Blood flow throughout the placenta decreases during contractions, which may result in metabolic decompensation of the fetus. Thus, it seems reasonable to recommend the use of continuous FHRM during labor in women with type 1 diabetes and IUGR from the onset of contractions [4]. Vaginal delivery of a fetus with umbilical artery AREDV is not recommended and CS is necessary even in very premature periods [4].

\section{Maternal Interventions and Preventive Strategies}

IUGR due to placental insufficiency is a progressive and irreversible disease. Except for intensive prenatal surveillance aimed at establishing appropriate timing of delivery, there are no perinatal interventions proven to be associated with improved outcomes of IUGR fetuses. Therefore, the question arises whether IUGR might be somehow prevented in women with type 1 diabetes. Although limited conclusions can be made regarding the prevention of IUGR in women with type 1 diabetes complicated by vasculopathy, pregnancy planning and appropriate management of vascular complications and hypertension could play an important role. While poor glycemic control in the second part of pregnancy stimulates fetal overgrowth, it can exert the opposite effects in early pregnancy by affecting early placental development. Evidence supporting this association comes from studies linking poor glycemic control in early pregnancy to preeclampsia $[72,73]$. 
IUGR is one of the major complications of preeclampsia, and there is a strong evidence that they share common pathophysiology [74-76]. Thus, interventions that have been found to reduce the risk of preeclampsia can also reduce the risk of IUGR. Low-dose aspirin $(50-150 \mathrm{mg})$ administered at or before 16 weeks of pregnancy has been found to reduce the risk of both [77]. However, there is no consensus on the use of aspirin for prevention of IUGR. The SOGC recommends lowdose aspirin use in high-risk patients, including women with pregestational diabetes and women with a previous history of IUGR or preeclampsia [5]; however, the ACOG and RCOG do not due to lack of evidence for its preventative effects for IUGR $[3,4]$. The ACOG and RCOG do, however, recommend its use in patients at high risk of preeclampsia. It is well known that the presence of even mild diabetic nephropathy is one of the strongest determinants of preeclampsia [22, 78, 79]. It is therefore reasonable to recommend low-dose aspirin from 12 gestational weeks for women with type 1 diabetes with vascular disease, primarily nephropathy, for the prevention of preeclampsia and associated complications, including IUGR [47•]. Moreover, early introduction of intensive antihypertensive treatment in women with type 1 diabetes with nephropathy may alleviate the severity of preeclampsia and increase the chances for normal fetal growth $[14,80]$. Treatment should be initiated when blood pressure (BP) exceeds 135/ $85 \mathrm{mmHg}$ or urinary albumin excretion exceeds $300 \mathrm{mg} /$ $24 \mathrm{~h}$. Less rigorous therapeutic targets ( $\mathrm{BP}>140 / 90 \mathrm{mmHg}$ or albuminuria $>2000 \mathrm{mg} / 24 \mathrm{~h}$ ) have been associated with an increased rate of preeclampsia and preterm delivery [80].

\section{Conclusion}

IUGR in pregnancy complicated by type 1 diabetes is usually the result of placental dysfunction related to maternal vasculopathy. Strict glycemic control and intensive hypertension treatment before and during pregnancy can potentially reduce the risk of IUGR in women with type 1 diabetes by improving placental function. Low-dose aspirin initiated at 12 weeks of gestation may be beneficial for women with type 1 diabetes at risk of IUGR, primarily those with diabetic nephropathy. Management of IUGR should focus on early diagnosis and intensive prenatal surveillance using ultrasound. Umbilical and uterine artery Doppler can be useful in the diagnosis and monitoring of fetuses with IUGR. Decisions regarding the timing of delivery should be based on the assessment of umbilical artery Doppler (the presence of AREDV). However, in fetuses with AREDV detected prior to 32 weeks, delivery should be considered when either DV abnormalities or UV pulsation is found. When preterm delivery is anticipated, antenatal steroids should be administered until $34+6$ weeks of gestation.

\section{Compliance with Ethical Standards}

Conflict of Interest Paweł Gutaj and Ewa Wender-Ożegowska declare that they have no conflict of interest.

Human and Animal Rights and Informed Consent This article does not contain any studies with human or animal subjects performed by any of the authors.

Open Access This article is distributed under the terms of the Creative Commons Attribution 4.0 International License (http:// creativecommons.org/licenses/by/4.0/), which permits unrestricted use, distribution, and reproduction in any medium, provided you give appropriate credit to the original author(s) and the source, provide a link to the Creative Commons license, and indicate if changes were made.

\section{References}

Papers of particular interest, published recently, have been highlighted as:

- Of importance

1. Barker DJ. Fetal origins of coronary heart disease. BMJ. 1995;311(6998):171-4.

2. Morgan AR, Thompson JM, Murphy R, Black PN, Lam WJ, Ferguson LR, et al. Obesity and diabetes genes are associated with being born small for gestational age: results from the Auckland Birthweight Collaborative study. BMC Med Genet. 2010;11:125. doi:10.1186/1471-2350-11-125.

3. ACOG. ACOG practice bulletin no. 134: fetal growth restriction. Obstet Gynecol. 2013;121(5):1122-33. doi:10.1097/01.AOG. 0000429658.85846.f9.

4. RCOG. Green-top guideline no. 31: the investigation and management of the small-for-gestational-age fetus. 2013.

5. Lausman A, Kingdom J, Gagnon R, Basso M, Bos H, Crane J, et al. Intrauterine growth restriction: screening, diagnosis, and management. J Obstet Gynaecol Can. 2013;35(8):741-57.

6. Figueras F, Gratacós E. Update on the diagnosis and classification of fetal growth restriction and proposal of a stage-based management protocol. Fetal Diagn Ther. 2014;36(2):86-98. This review provides practical stage-based approach to the management of IUGR.

7. Berk MA, Mimouni F, Miodovnik M, Hertzberg V, Valuck J. Macrosomia in infants of insulin-dependent diabetic mothers. Pediatrics. 1989;83(6):1029-34.

8. Kyne-Grzebalski D, Wood L, Marshall SM, Taylor R. Episodic hyperglycaemia in pregnant women with well-controlled type 1 diabetes mellitus: a major potential factor underlying macrosomia. Diabet Med. 1999;16(8):702-6.

9. Wender-Ozegowska E, Gutaj P, Szczepanek U, Ozegowska K, Zawiejska A, Brazert J. Influence of pregnancy planning on obstetrical results in women with pregestational diabetes mellitus. Ginekol Pol. 2010;81(10):762-7.

10. Scifres CM, Feghali MN, Althouse AD, Caritis SN, Catov JM. Effect of excess gestational weight gain on pregnancy outcomes in women with type 1 diabetes. Obstet Gynecol. 2014;123(6): 1295-302. doi:10.1097/AOG.0000000000000271.

11. Secher AL, Parellada CB, Ringholm L, Asbjornsdottir B, Damm P, Mathiesen ER. Higher gestational weight gain is associated with increasing offspring birth weight independent of maternal glycemic 
control in women with type 1 diabetes. Diabetes Care. 2014;37(10): 2677-84. doi:10.2337/dc14-0896.

12. Gutaj P, Sawicka-Gutaj N, Brazert M, Wender-Ozegowska E. Insulin resistance in pregnancy complicated by type 1 diabetes mellitus. Do we know enough? Ginekol Pol. 2015;86(3):219-23.

13. Gutaj P, Wender-Ozegowska E, Iciek R, Zawiejska A, Pietryga M, Brazert J. Maternal serum placental growth factor and fetal SGA in pregnancy complicated by type 1 diabetes mellitus. J Perinat Med. 2014;42(5):629-33. doi:10.1515/jpm-2013-0227.

14. Reece EA, Leguizamon G, Homko C. Stringent controls in diabetic nephropathy associated with optimization of pregnancy outcomes. J Matern Fetal Med. 1998;7(4):213-6. doi:10.1002/(SICI)15206661(199807/08)7:4<213::AID-MFM11>3.0.CO;2-E.

15. Piccoli GB, Clari R, Ghiotto S, Castelluccia N, Colombi N, Mauro $\mathrm{G}$, et al. Type 1 diabetes, diabetic nephropathy, and pregnancy: a systematic review and meta-study. Rev Diabet Stud. 2013;10(1):626. doi:10.1900/RDS.2013.10.6.

16. Pambianco G, Costacou T, Ellis D, Becker DJ, Klein R, Orchard TJ. The 30-year natural history of type 1 diabetes complications: the Pittsburgh Epidemiology of Diabetes Complications Study experience. Diabetes. 2006;55(5):1463-9. doi:10.2337/db05-1423.

17. Maahs DM, West NA, Lawrence JM, Mayer-Davis EJ. Chapter 1: epidemiology of type 1 diabetes. Endocrinol Metab Clin North Am. 2010;39(3):481-97. doi:10.1016/j.ecl.2010.05.011.

18. Desoye G, Shafrir E. Placental metabolism and its regulation in health and diabetes. Mol Aspects Med. 1994;15(6):505-682.

19. Redline RW, Boyd T, Campbell V, Hyde S, Kaplan C, Khong TY, et al. Maternal vascular underperfusion: nosology and reproducibility of placental reaction patterns. Pediatr Dev Pathol. 2004;7(3):23749. doi:10.1007/s10024-003-8083-2.

20. Starikov R, Inman K, Chen K, Lopes V, Coviello E, Pinar H, et al. Comparison of placental findings in type 1 and type 2 diabetic pregnancies. Placenta. 2014;35(12):1001-6. doi:10.1016/j. placenta.2014.10.008

21. Stevens DU, Al-Nasiry S, Bulten J, Spaanderman MEA. Decidual vasculopathy in preeclampsia: Lesion characteristics relate to disease severity and perinatal outcome. 2013. p. 805-9.

22. Jensen DM, Damm P, Ovesen P, Molsted-Pedersen L, BeckNielsen H, Westergaard JG, et al. Microalbuminuria, preeclampsia, and preterm delivery in pregnant women with type 1 diabetes: results from a nationwide Danish study. Diabetes Care. 2010;33(1):90-4. doi:10.2337/dc09-1219.

23. Fitzgerald B, Kingdom J, Keating S. Distal villous hypoplasia. Diagn Histopathol. 2012;18(5):195-200. doi:10.1016/j.mpdhp. 2012.02.005.

24. Ogino S, Redline RW. Villous capillary lesions of the placenta: distinctions between chorangioma, chorangiomatosis, and chorangiosis. Hum Pathol. 2000;31(8):945-54. doi:10.1053/hupa. 2000.9036.

25. Salafia CM, Silberman L. Placental pathology and abnormal fetal heart rate patterns in gestational diabetes. Pediatr Pathol. 1989;9(5): 513-20.

26. Holcberg G, Huleihel M, Sapir O, Katz M, Tsadkin M, Furman B, et al. Increased production of tumor necrosis factor-alpha TNF-alpha by IUGR human placentae. Eur J Obstet Gynecol Reprod Biol. 2001;94(1):69-72.

27. Bartha JL, Romero-Carmona R, Comino-Delgado R. Inflammatory cytokines in intrauterine growth retardation. Acta Obstet Gynecol Scand. 2003;82(12):1099-102.

28. Clausen P, Ekbom P, Damm P, Feldt-Rasmussen U, Nielsen B, Mathiesen ER, et al. Signs of maternal vascular dysfunction precede preeclampsia in women with type 1 diabetes. J Diabetes Complications. 2007;21(5):288-93. doi:10.1016/j.jdiacomp.2006. 03.004 .

29. Salafia CM, Pezzullo JC, Minior VK, Divon MY. Placental pathology of absent and reversed end-diastolic flow in growth-restricted fetuses. Obstet Gynecol. 1997;90(5):830-6. doi:10.1016/S00297844(97)00473-0.

30. Madazli R, Somunkiran A, Calay Z, Ilvan S, Aksu MF. Histomorphology of the placenta and the placental bed of growth restricted foetuses and correlation with the Doppler velocimetries of the uterine and umbilical arteries. Placenta. 2003;24(5):510-6.

31. Aardema MW, Oosterhof H, Timmer A, van Rooy I, Aarnoudse JG. Uterine artery Doppler flow and uteroplacental vascular pathology in normal pregnancies and pregnancies complicated by preeclampsia and small for gestational age fetuses. Placenta. 2001;22(5):405-11. doi:10.1053/plac.2001.0676.

32. Pietryga M, Brazert J, Wender-Ozegowska E, Biczysko R, Dubiel $\mathrm{M}$, Gudmundsson S. Abnormal uterine Doppler is related to vasculopathy in pregestational diabetes mellitus. Circulation. 2005;112(16):2496-500. doi:10.1161/CIRCULATIONAHA.104. 492843.

33. Bennett SN, Tita A, Owen J, Biggio JR, Harper LM. Assessing White's classification of pregestational diabetes in a contemporary diabetic population. Obstet Gynecol.125(5):1217-23. doi:10.1097/ AOG.0000000000000820

34. Salvesen DR, Freeman J, Brudenell JM, Nicolaides KH. Prediction of fetal acidaemia in pregnancies complicated by maternal diabetes mellitus by biophysical profile scoring and fetal heart rate monitoring. BJOG. 1993;100(3):227-33. doi:10.1111/j.1471-0528.1993. tb15235.x.

35. Persson M, Pasupathy D, Hanson U, Norman M. Birth size distribution in 3,705 infants born to mothers with type 1 diabetes: a population-based study. Diabetes Care. 2011;34(5):1145-9. doi: $10.2337 / \mathrm{dc} 10-2406$.

36. Bradley RJ, Nicolaides KH, Brudenell JM. Are all infants of diabetic mothers "macrosomic"? BMJ. 1988;297(6663):1583-4.

37. Johnstone FD, Mao JH, Steel JM, Prescott RJ, Hume R. Factors affecting fetal weight distribution in women with type I diabetes. BJOG. 2000;107(8):1001-6.

38. Howarth C, Gazis A, James D. Associations of Type 1 diabetes mellitus, maternal vascular disease and complications of pregnancy. Diabet Med. 2007;24(11):1229-34. doi:10.1111/j.1464-5491.2007. 02254.x.

39. Reece EA, Hagay Z, Assimakopoulos E, Moroder W, Gabrielli S, DeGennaro N, et al. Diabetes mellitus in pregnancy and the assessment of umbilical artery waveforms using pulsed Doppler ultrasonography. J Ultrasound Med. 1994;13(2):73-80.

40. Strotmeyer ES, Steenkiste AR, Foley TP, Berga SL, Dorman JS, Menstrual cycle differences between women with type 1 diabetes and women without diabetes. Diabetes Care. 2003;26(4):1016-21. doi:10.2337/diacare.26.4.1016.

41. Schweiger BM, Snell-Bergeon JK, Roman R, McFann K, Klingensmith GJ. Menarche delay and menstrual irregularities persist in adolescents with type 1 diabetes. Reprod Biol Endocrinol. 2011;9:61. doi:10.1186/1477-7827-9-61.

42. Papageorghiou AT, Kennedy SH, Salomon LJ, Ohuma EO, Cheikh Ismail L, Barros FC, et al. International standards for early fetal size and pregnancy dating based on ultrasound measurement of crownrump length in the first trimester of pregnancy. Ultrasound Obstet Gynecol. 2014;44(6):641-8. doi:10.1002/uog.13448.

43. Villar J, Papageorghiou AT, Pang R, Ohuma EO, Ismail LC, Barros $\mathrm{FC}$, et al. The likeness of fetal growth and newborn size across nonisolated populations in the INTERGROWTH-21st Project: the Fetal Growth Longitudinal Study and Newborn Cross-Sectional Study. Lancet Diab Endocrinol. 2014;2(10):781-92. doi:10.1016/ s2213-8587(14)70121-4.

44. Price JH, Hadden DR, Archer DB, Harley JM. Diabetic retinopathy in pregnancy. Br J Obstet Gynaecol. 1984;91(1):11-7.

45. Klein BE, Klein R, Meuer SM, Moss SE, Dalton DD. Does the severity of diabetic retinopathy predict pregnancy outcome? J Diabet Complications. 1988;2(4):179-84. 
46. Reece EA, Sivan E, Francis G, Homko CJ. Pregnancy outcomes among women with and without diabetic microvascular disease (White's classes B to FR) versus non-diabetic controls. Am J Perinatol. 1998;15(9):549-55. doi:10.1055/s-2007-994059.

47. Mathiesen ER, Ringholm L, Feldt-Rasmussen B, Clausen P, Damm P. Obstetric nephrology: pregnancy in women with diabetic nephropathy - the role of antihypertensive treatment. Clin J Am Soc Nephrol. 2012;7(12):2081-8. doi:10.2215/cjn.00920112.

48. Khoury MJ, Erickson JD, Cordero JF, McCarthy BJ. Congenital malformations and intrauterine growth retardation: a population study. Pediatrics. 1988;82(1):83-90.

49. Reece EA. Diabetes-induced birth defects: what do we know? What can we do? Curr Diab Rep. 2012;12(1):24-32. doi:10.1007/ s11892-011-0251-6.

50. M. Kady S, Gardosi J. Perinatal mortality and fetal growth restriction. Best Pract Res Clin Obstet Gynaecol. 2004;18(3):397-410. doi:10.1016/j.bpobgyn.2004.02.009.

51. Blair EM, Nelson KB. Fetal growth restriction and risk of cerebral palsy in singletons born after at least 35 weeks gestation. Am J Obstet Gynecol. 2015;212(4):520 e1-7. doi:10.1016/j.ajog.2014. 10.1103 .

52. Reece EA, Smikle C, O'Connor TZ, Holford T, Nelson-Robinson $\mathrm{L}$, Degennaro N, et al. A longitudinal study comparing growth in diabetic pregnancies with growth in normal gestations: I. The fetal weight. Obstet Gynecol Surv. 1990;45(3):161-4.

53. Morris RK, Meller CH, Tamblyn J, Malin GM, Riley RD, Kilby $\mathrm{MD}$, et al. Association and prediction of amniotic fluid measurements for adverse pregnancy outcome: systematic review and metaanalysis. BJOG. 2014;121(6):686-99. doi:10.1111/1471-0528. 12589.

54. Nabhan AF, Abdelmoula YA. Amniotic fluid index versus single deepest vertical pocket as a screening test for preventing adverse pregnancy outcome. Cochrane Database Syst Rev. 2008(3): CD006593. doi:10.1002/14651858.CD006593.pub2

55. Bracero LA, Figueroa R, Byrne DW, Han HJ. Comparison of umbilical Doppler velocimetry, nonstress testing, and biophysical profile in pregnancies complicated by diabetes. J Ultrasound Med. 1996;15(4):301-8.

56. Dicker D, Feldberg D, Yeshaya A, Peleg D, Karp M, Goldman JA. Fetal surveillance in insulin-dependent diabetic pregnancy: predictive value of the biophysical profile. Am J Obstet Gynecol. 1988;159(4):800-4.

57. Kaplan M. Fetal breathing movements. An update for the pediatrician. Am J Dis Child. 1983;137(2):177-81.

58. Bar-Hava I, Scarpelli SA, Barnhard Y, Divon MY. Amniotic fluid volume reflects recent glycemic status in gestational diabetes mellitus. Am J Obstet Gynecol. 1994;171(4):952-5.

59. Edelberg SC, Dierker L, Kalhan S, Rosen MG. Decreased fetal movements with sustained maternal hyperglycemia using the glucose clamp technique. Am J Obstet Gynecol. 1987;156(5):1101-5.

60. Reece EA, Hagay Z, Roberts AB, DeGennaro N, Homko CJ, Connolly-Diamond $\mathrm{M}$, et al. Fetal Doppler and behavioral responses during hypoglycemia induced with the insulin clamp technique in pregnant diabetic women. Am J Obstet Gynecol. 1995; 172(1 Pt 1):151-5.

61. Froen JF, Tveit JV, Saastad E, Bordahl PE, Stray-Pedersen B, Heazell AE, et al. Management of decreased fetal movements. Semin Perinatol. 2008;32(4):307-11. doi:10.1053/j.semperi.2008. 04.015.

62. Kitzmiller JL, Block JM, Brown FM, Catalano PM, Conway DL, Coustan DR, et al. Managing preexisting diabetes for pregnancy: summary of evidence and consensus recommendations for care. Diabetes Care. 2008;31(5):1060-79. doi:10.2337/dc08-9020.

63. Gulmezoglu AM, Hofmeyr GJ. Bed rest in hospital for suspected impaired fetal growth. Cochrane Database Syst Rev. 2000;2: CD000034. doi:10.1002/14651858.CD000034.
64. Langer O, Levy J, Brustman L, Anyaegbunam A, Merkatz R, Divon M. Glycemic control in gestational diabetes mellitus - how tight is tight enough: small for gestational age versus large for gestational age? Am J Obstet Gynecol. 1989;161(3):646-53.

65. Parikh RM, Joshi SR, Menon PS, Shah NS. Intensive glycemic control in diabetic pregnancy with intrauterine growth restriction is detrimental to fetus. Med Hypotheses. 2007;69(1):203-5. doi: 10.1016/j.mehy.2006.10.020.

66. The Guideline Development G. Management of diabetes from preconception to the postnatal period: summary of NICE guidance. BMJ. 2008;336(7646):714-7. doi:10.1136/bmj.39505.641273.AD.

67. Roberts D, Dalziel S. Antenatal corticosteroids for accelerating fetal lung maturation for women at risk of preterm birth. Cochrane Database Syst Rev. 2006(3):CD004454. doi:10.1002/14651858. CD004454.pub2

68. Mathiesen ER, Christensen AB, Hellmuth E, Hornnes P, Stage E, Damm P. Insulin dose during glucocorticoid treatment for fetal lung maturation in diabetic pregnancy: test of an algorithm [correction of analgoritm]. Acta Obstet Gynecol Scand. 2002;81(9):835-9.

69. Kaushal K, Gibson JM, Railton A, Hounsome B, New JP, Young RJ. A protocol for improved glycaemic control following corticosteroid therapy in diabetic pregnancies. Diabet Med. 2003;20(1):73-5.

70. Berkley E, Chauhan SP, Abuhamad A. Doppler assessment of the fetus with intrauterine growth restriction. Am J Obstet Gynecol. 2012;206(4):300-8. doi:10.1016/j.ajog.2012.01.022.

71. Baschat AA, Cosmi E, Bilardo CM, Wolf H, Berg C, Rigano S, et al. Predictors of neonatal outcome in early-onset placental dysfunction. Obstet Gynecol. 2007;109(2 Pt 1):253-61. doi:10.1097/01. AOG.0000253215.79121.75.

72. Ekbom P, Damm P, Nogaard K, Clausen P, Feldt-Rasmussen U, Feldt-Rasmussen B, et al. Urinary albumin excretion and 24-hour blood pressure as predictors of pre-eclampsia in type I diabetes. Diabetologia. 2000;43(7):927-31.

73. Hanson U, Persson B. Epidemiology of pregnancy-induced hypertension and preeclampsia in type 1 (insulin-dependent) diabetic pregnancies in Sweden. Acta Obstet Gynecol Scand. 1998;77(6): $620-4$.

74. Khong TY, De Wolf F, Robertson WB, Brosens I. Inadequate maternal vascular response to placentation in pregnancies complicated by pre-eclampsia and by small-for-gestational age infants. $\mathrm{Br} \mathrm{J}$ Obstet Gynaecol. 1986;93(10):1049-59.

75. Rasmussen S, Irgens LM, Albrechtsen S, Dalaker K. Predicting preeclampsia in the second pregnancy from low birth weight in the first pregnancy. Obstet Gynecol. 2000;96(5 Pt 1):696-700.

76. Rasmussen S, Irgens LM. History of fetal growth restriction is more strongly associated with severe rather than milder pregnancyinduced hypertension. Hypertension. 2008;51(4):1231-8. doi:10. 1161/HYPERTENSIONAHA.107.096248.

77. Bujold E, Roberge S, Lacasse Y, Bureau M, Audibert F, Marcoux S, et al. Prevention of preeclampsia and intrauterine growth restriction with aspirin started in early pregnancy: a meta-analysis. Obstet Gynecol. 2010;116(2 Pt 1):402-14. doi:10.1097/AOG. 0b013e3181e9322a.

78. Sibai BM, Caritis S, Hauth J, Lindheimer M, VanDorsten JP, MacPherson C, et al. Risks of preeclampsia and adverse neonatal outcomes among women with pregestational diabetes mellitus. National Institute of Child Health and Human Development Network of Maternal-Fetal Medicine Units. Am J Obstet Gynecol. 2000;182(2):364-9.

79. Combs CA, Rosenn B, Kitzmiller JL, Khoury JC, Wheeler BC, Miodovnik M. Early-pregnancy proteinuria in diabetes related to preeclampsia. Obstet Gynecol. 1993;82(5):802-7.

80. Nielsen LR, Damm P, Mathiesen ER. Improved pregnancy outcome in type 1 diabetic women with microalbuminuria or diabetic nephropathy: effect of intensified antihypertensive therapy? Diabetes Care. 2009;32(1):38-44. doi:10.2337/dc08-1526. 